\title{
Molecular and Classical Cytogenetic Analyses Demonstrate an Apomorphic Reciprocal Chromosomal Translocation in Gorilla gorilla
}

\author{
R. STANYON, J. WIENBERG, D. ROMAGNO, F. BIGONI, A. JAUCH, \\ AND T. CREMER \\ Istituto di Antropologia fisica, University of Genoa, 16126 Genoa, Italy \\ (R.S., F.B.); Institut für Humangenetik und Anthropologie, University of \\ Heidelberg, D-6900 Heidelberg, Germany (J.W., A.J., T.C.); Istituto di \\ Antropologia, University of Florence, Florence, Italy (D.R.)
}

KEY WORDS In situ hybridization, chromosome banding, phylogeny, primates

\begin{abstract}
The existence of an apomorphic reciprocal chromosomal translocation in the gorilla lineage has been asserted or denied by various cytogeneticists. We employed a new molecular cytogenetic strategy (chromosomal in situ suppression hybridization) combined with high-resolution banding, replication sequence analysis, and fluorochrome staining to demonstrate that a reciprocal translocation between ancestral chromosomes homologous to human chromosome 5 and 17 has indeed occurred. (c) 1992 Wiley-Liss, Inc
\end{abstract}

Comparative studies of banded chromosomes in primates have demonstrated that particular chromosomal mutations, or rearrangements, are often characteristic of different clades. For example, while fusions characterize the prosimians, fissions are common in the evolution of guenons, and inversions (especially pericentric inversions) are thought to account for the majority of chromosomal changes in the hominoids. However, Dutrillaux $(1973,1975)$ and later Yunis and Prakash (1982) pointed out the possibility of a reciprocal translocation in the gorilla lineage between chromosomes homologous to human chromosomes 5 and 17. This rearrangement was not mentioned by other cytogeneticists interested in human and ape evolution (Seuánez, 1979; Stanyon and Chiarelli, 1982). Marks (1982) has suggested that these conflicting reports are due to problems of precision of resolution in cytogenetic studies. Seuánez (1987:72), however, provided a more definitive statement

"Though the pattern of chromosome banding might give partial support to this presumptive rearrangement, comparative gene assignment of the thymidine kinase locus
(TK) has ruled out the possibility that such rearrangement has ever occurred."

In making this statement Seuánez was following the normal procedure because gene mapping is usually given priority in establishing homologies (Ruddle, 1981). However, gene mapping is not always a foolproof indication of homology. For example, genes mapped to human $2 p$ and $2 q$ are found on the "wrong" great ape arm homologs (Seuanez, 1987). A conflict also exists for the inosine-triphosphatase (ITPA) locus. This gene is mapped to human 20, but it is not on the seemingly identical great ape chromosome (O'Brien and Graves, 1990). Perhaps, then, TK1 gene assignment has not definitively ruled out the existence of an apomorphic 5/17 translocation in the gorilla lineage and this possibility could be profitably reexamined.

We decided to study this region systematically using high resolution banding techniques (Yunis, 1981), fluorochrome staining (Schmid et al., 1986; Wienberg and Stanyon,

Received May 14, 1991; accepted October 30, 1991 
1987, 1988), replication banding (Weber et al., 1986, Stanyon et al., 1990) and in situ suppression (CISS) hybridization (Lichter et al., 1988; Pinkel et al., 1988) of human DNA libraries to gorilla chromosomes and to the chromosomes of other primate species. These data amply demonstrate that a 5/17 translocation occurred in the gorilla lineage.

\section{MATERIAL AND METHODS}

Chromosome preparations were made from both phytohemagglutinin stimulated whole blood and fibroblast cultures of human, chimpanzee, gorilla, and macaque (Macaca fuscata). High resolution G-banding and replication banding were done as reported before (Small et al., 1985; Stanyon et al., 1990). Distamycin/DAPI staining was according to Wienberg and Stanyon (1988).

CISS hybridization to primate chromosomes was as described previously (Wienberg et al., 1990). Briefly, chromosome specific human DNA libraries established from flow sorted chromosomes 5 and 17 and cloned to phages (ATCC Nr. LA05NS01, LL17NS02) or plasmids (chromosome $17 \mathrm{li}$ brary kindly provided by J. Gray, Livermore, CA, U.S.A.) were used as probes. Slides were mounted with $18 \mathrm{~mm} \times 18 \mathrm{~mm}$ cover slips and standard hybridization mixture with $2 \mu \mathrm{g}$ labelled phage library DNA or 300 microgram labelled plasmid library DNA. Labelling was by standard nick-translocation protocol. Probes were labelled with biotin and detected with fluorescein (FITC) coupled to avidin (Vector Laboratories), diluted $1: 1000$ in TE. Unlabelled human competitor DNA and salmon sperm carrier DNA was as described (Lichter et al., 1988).

Double hybridization experiments were performed with digoxigenin labelled chromosome 17 probe, which was detected with FITC labelled mouse anti-digoxigenin antibodies (Boehringer Mannheim) diluted $1: 1000$ in $\mathrm{TE}$, together with biotin labelled human 5 probe that was detected with avidin coupled to AMCA (Vector Laboratories), diluted 1:200 in TE. AMCA signals were amplified once as described by Pinkel et al. (1986) and analysed with the same filter combination as for DAPI. Slides were analysed with a Zeiss photomicroscope III equipped for FITC and DAPI fluorescence.

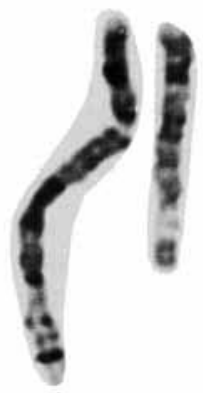

A

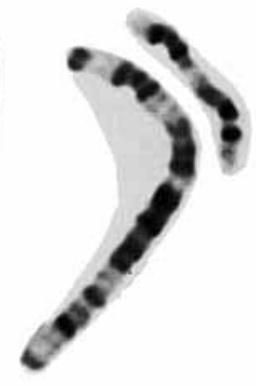

B
Fig. 1. From left to right: a high resolution G-band comparison of human chromosomes 5 and gorilla 19 , and gorilla 4 and human 17. Human 17 has been in verted to better demonstrate the banding homology. Note the apomorphic teleomeric heterochromatin in the gorilla chromosomes.

Photographs were taken on AGFA-CHROM 1000 RS or Kodak T-MAX black and white (400 ASA) films.

\section{RESULTS}

High resolution G-banding clearly showed a striking correspondence of banding between the short arm and a part of the long arm of gorilla 4 and the long arm of human 17. There was an equally good match of banding between the short arm and part of the long arm of human 5 and most of gorilla 19 (Fig. 1). Additional, terminal G-bands were noted in gorilla 4 and gorilla 19 .

The replication sequence demonstrated that the similarity in banding pattern extended to the DNA replication timing of the G-bands (Fig. 2). In particular, gorilla 19 has a large band on the short arm which is late replicating not only within the chromosome, but also one of the latest-replicating regions within the entire karyotype. This replication timing is identical to that of band 5 p14 in humans. The replication order of gorilla $4 p$ corresponds to the replication order of human 17q. However, the replication order of the terminal half of gorilla $19 q$ had no obvious counterpart in the human karyotype. The gorilla chromosomes had late teleomeric replicating heterochromatin not present in humans.

DA/DAPI staining showed that the distribution of brilliant fluorescent bands in all species was as previously reported (Schmid 


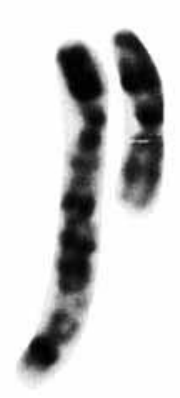

A

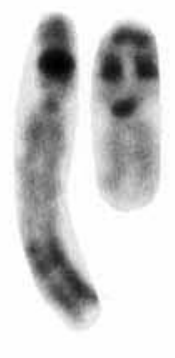

B

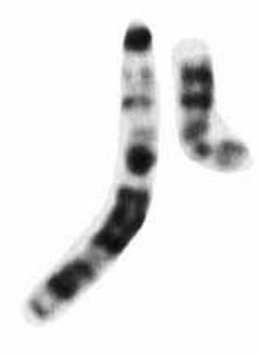

C

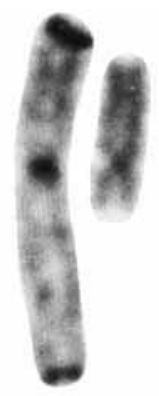

D

Fig. 2. A and B: The replication pattern of human 5 and gorilla 19 . A, complete replication; B, late replication pattern; $C$ and $D$ : The replication pattern of gorilla 4 and human 17 (inverted to better demonstrate the replication homology). C, complete replication; D, late replication pattern. Note the additional late replicating teleomeric heterochromatin in the gorilla.

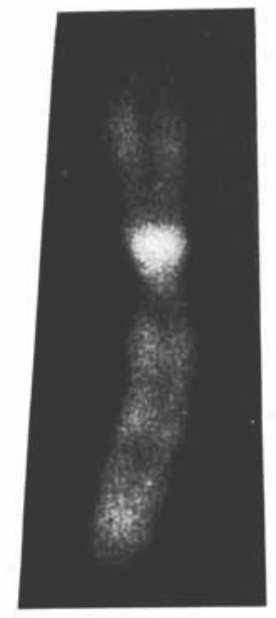

Fig. 3. Shows the DA/DAPI fluorescent band on gorilla 4 . This DA/DAPI band was most probably translocated from an ancestral chromosome homologous to human 17 (see text).

et al., 1986; Wienberg and Stanyon, 1987, 1988). Relevant to our analysis, a pericentromeric bright band was found on the gorilla $4 \mathrm{p}$, and chimpanzee 19 , but not on human 5, chimpanzee 4, or gorilla 19 (Fig. 3). The macaque chromosome had no DADAPI brilliant bands.

The DNA libraries of human 5 and 17 each hybridized to one chromosome pair in humans, chimpanzees, and macaques. In the gorilla each probe hybridized to four chromosomes (Fig. 4). Human 17 libraries hybridized to the entire short arm and a part of the long arm next to the centromere of gorilla 4 and to about half of the long arm of gorilla 19 (Fig. 4c). The human 5 library hybridized to the short arm, to half of the long arm adjacent to the centromere of gorilla 19, and to most of the long arm of gorilla 4 (Fig. 4d). In gorilla chromosomes, late replicating teleomeric C-bands were not hybridized with either chromosome 5 or 17 libraries.

\section{DISCUSSION}

The hybridization data alone demonstrate that at the molecular level there can be no doubt that a reciprocal translocation occurred in the gorilla lineage between homologs to human 5 and 17 (Fig. 5). The highresolution replication sequence and DA DAPI banding also clearly show that a reciprocal translocation has indeed occurred. The comparison with outgroup chromosomes, from $M$. fuscata, indicates that this reciprocal translocation in the gorilla is apomorphic.

According to Wienberg and Stanyon (1988), the last hominoid common ancestor had a DA/DAPI band on the homolog equivalent to human 17 . The gorilla lacks a DA DAPI band on this chromosome and is the only hominoid to have such a bright band on the homolog to human 5. It is most likely that this DA/DAPI band was derived from an ancestral homolog to human 17 and is additional evidence for the reciprocal trans- 

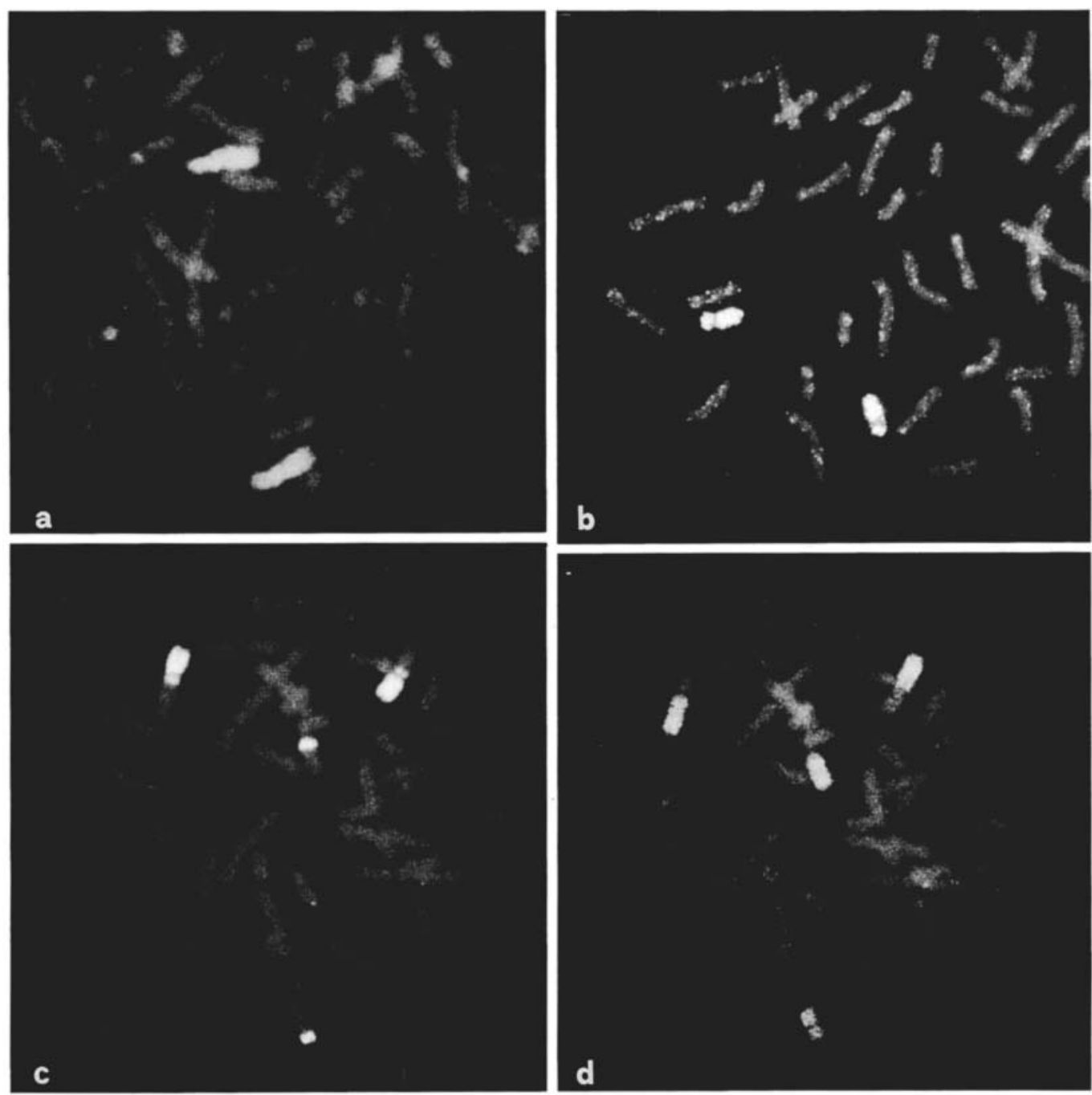

Fig. 4. CISS hybridization patterns of human 5 and 17 libraries to primate chromosomes. Hybridization was detected either with fluorescein (FITC) (a, b, and c) or AMCA (d). Chromosomes were counterstained with propidiumiodine. a: Chromosome phage 17 library hybridized to chimpanzee chromosomes. One pair of chromosomes is hybridized. Note the slight cross hybridization to the heterochromatin in some centromeres due to sequence homology. b: Chromosome plasmid 17 library on $M$. fuscata chromosomes, as in the chimpanzee one chro-

mosome pair is hybridized. $c$ and $d$ : The same gorilla metaphase hybridized with human 17 plasmid library (c) which was labelled with digoxigenin and detected with FITC labelled mouse anti-bodies, and human $5 \mathrm{ph}$ age library (d) labelled with biotin and detected with avidin coupled to AMCA. This procedure allows two different probes to be visualized on the same metaphase in different colors by just changing the filter combination of the microscope. With both probes, four chromosomes were labelled, demonstrating the reciprocal translocation.

location, even if the molecular basis and significance of DA/DAPI is not yet known.

It is clear that the reciprocal translocation was not a simple exchange of arms, because the hybridization signals of each probe are

found on both the short and the long arm of different chromosomes. Consequently, the short arm, the centromere, and a small proximal part of the long arm of gorilla 4 is homologous to human 17 , while the part of go- 


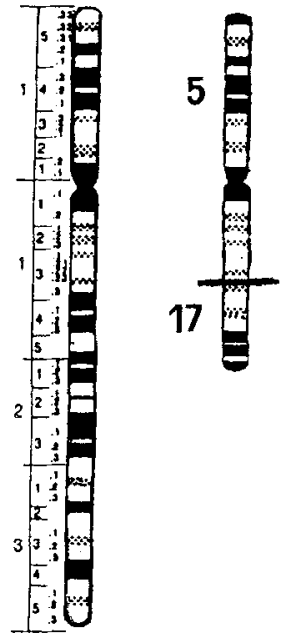

5

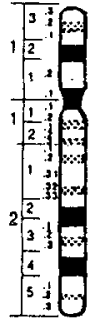

17

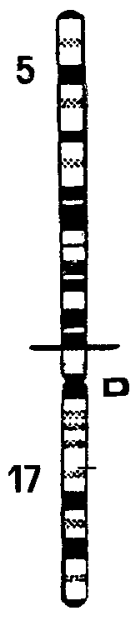

Fig. 5. Idiograms of, from left to right, human chromosome 5, gorilla 19 , human 17 , and gorilla 4 . Human chromosomes are numbered below. The gorilla chromosomes are numbered on the left to summarize the hybridization pattern obtained with human probes. Gorilla chromosome 4 (extreme right) is inverted to better show the homology to human 17 . The $\mathrm{D}$ to the right of gorilla chromosome 4 indicates the location of the DA DAPI bright band.

rilla 19 homologous to human 5 includes the short arm, the centromere, and the proximal half of the long arm. It appears that the centromere of gorilla 4 comes from an ancestral chromosome homologous to human 17 , while the centromere of gorilla 19 derives from an ancestral chromosome homologous to human 5 .

There are several possibilities to explain the inconsistency between chromosomal homology and gene mapping. TK1, gene assignment in the gorilla, could be erroneous. Chromosomal rearrangements in vitro in hybrid cell lines are probably more common than previously thought, and the analysis of hybrid cell chromosomes by banding can be difficult. On the other hand, there may be a small, undetected rearrangement which has altered the chromosomal location of TK1 in the gorilla. Notably, there is no good correspondence between either the high-resolution banding or replication sequence of the terminal half of gorilla 19 and any part of human 17. CISS hybridization shows that this chromosome section derives from a homolog to human 17, but the lack of banding correspondence suggests that it has been subject to further, as yet not clear, alterations. Further study with molecular cytogenetic techniques could clarify whether or not such rearrangements have involved the TK1 gene.

CISS hybridization, in contrast to single gene mapping, signals the presence of all parts of the homologous chromosome in the target species, regardless of where they are. Single genes, however, are localized to specific chromosomal regions, and chromosomal homologies between mapped genes are interpolations. There is no guarantee that chromosomal regions between markers are actually homologous.

A limitation of using whole chromosome probes is that they do not allow the mapping of intrachromosomal rearrangements. Microdissection and microcloning of chromosome bands, YAC (yeast artificial chromosomes) and cosmid clones, and pools of single copy sequences can provide probes for chromosome subregions from any species (Ajioka et al., 1990; Wienberg et al., 1990; Arnold et al., 1991; Lengauer et al., 1991). CISS hybridization was recently used to map more complex DNA probes on a single human chromosome (Lichter et al., 1990), then the maximum number of genes (44) that have been mapped to all the chromosomes of the gorilla (O'Brien and Graves, 1990). CISS hybridization, combined with traditional chromosome techniques, will permit a new level of confidence and utility for reconstructing and clarifying primate evolution and human origins.

\section{ACKNOWLEDGMENTS}

We would like to thank A. Wiegenstein and J. Hollatz for photographic work. T.C. was the recipient of a Heisenberg-Stipendium from the Deutsche Forschungsgemeinschaft. This work was supported in part by the Deutsche Forschungsgemeinschaft and the Italian Ministry of Universities and Scientific Research, $60 \%$ and $40 \%$ grants, and CNR grant number 90.2476.CT04.

\section{LITERATURE CITED}

Arnold N, Wienberg J, Zachau H-G (1991) Evolution of V-kappa immunoglobulin genes in human and pri- 
mates analysed by molecular cytogenetics (abstract). Am. J. Hum. Genet. (in press).

Ajioka JW, Garza D, Johnson D, Carulli JP, Jones RW, and Hartl DL (1990) Genome evolution analyzed by cloning large fragments of Drosophila DNA in yeast artificial chromosomes. In MT Cleg and SJ O'Brien (eds.): Molecular Evolution. New York: Wiley-Liss, pp. 253-263.

Dutrillux B (1975) Sur le nature et l'origine des chromosomes humanines. Paris:L'expansion Scientifique.

Dutrillaux B, Rethoré M-O, Prieur M, and Lejeune J (1973) Analyse de la structure fine de chromosomes du Gorille (Gorilla gorilla). Hum. Genet. 20:343-354.

Lengauer C, Lüdecke H-J, Wienberg J, Cremer T, and Horsthemke B (1991) Comparative chromosome band mapping in primates by in situ suppression hybridization of band specific DNA microlibraries. Hum. Evol. 6:67-71.

Lichter P, Cremer T, Broden J, Manuelidis L, and Ward DC (1988) Delineation of individual human chromosomes in metaphase and interphase cells by in situ suppression hybridization using recombinant DNA libraries. Hum. Genet 80:224-234.

Lichter P, Chang Tang CJ, Call K, Hermanson G, Evans GA, Housman D, and Ward DC (1990) High resolution mapping of human chromosome 11 by in situ hybridization with cosmid clones. Science 247:64-69.

Marks J (1983) Hominoid cytogenetics and Evolution. Year-book Phys. Anthropol. 26:131-159.

O'Brien SJ, Seuánez HN, and Womack JE (1988) Mammalian genome organization: an evolutionary view. Annu. Rev. Genet. 22:323-351.

O'Brien SJ, and Graves JA (1990) Report of the committee on comparative gene mapping (HGM10.5). Cytogen. Cell Genet. 55:406-433.

Pinkel D, Gray JW, Trask B, Engh G van den, Fuscoe J, Dekken $H$ van (1986) Cytogenetic analysis by in situ hybridization with fluorescently labelled nucleic acid probes. Cold Spring Harb. Symp. Quant. Biol. 51:151157.
Pinkel D, Landegent J, Collins C, Fuscoe J, Segraves R, Lucas J, and Gray JW (1988) Fluorescence in situ hybridization with human chromosome specific libraries: Detection of trisomy 21 and translocations of chromosome 4. Proc. Natl. Acad. Sci. U.S.A. 85:91389142.

Ruddle FH (1981) A new era in mammalian gene mapping: Somatic cell genetics and recombinant DNA methodologies. Nature 294:115-119.

Schmid M, Haaf T, Ott G, Scheres JMJC, and Wensing JAB (1986) Heterochromatin in the chromosomes of the gorilla: Characterization with distamycin A/DAPI, D287/170, chromomycin A3, quinacrine, and 5-azacytidine. Cytogenet. Cell Genet. 41:71-82.

Stanyon R, Ramagno D, Wienberg J, and Maurer U (1990) Sequence of DNA replication in Macaca fuscata chromosomes: An outgroup for phylogenetic comparison between man and apes. Genetica 80:45-52.

Wever B, Schempp W, and Wiesner H (1986) An evolutionarily conserved early replicating segment on the sex chromosomes of man and the great apes. Cytogenet. Cell Genet. 43:72-78.

Wienberg J, Jauch A, Stanyon R, and Cremer T (1990) Molecular cytotaxonomy of primates by chromosomal in situ suppression hybridization. Genomics 8:347350 .

Wienberg J, and Stanyon R (1987) Fluorescent heterochromatin staining in primate chromosomes. Human Evol. 2:445-457.

Wienberg $J$, and Stanyon $R$ (1988) DA/DAPI fluorescent bands in the chromosomes of Pan paniscus. Am. J. Primatol. 14:91-96.

Yunis JJ (1981) Mid-prophase human chromosomes. The attainment of 2000 bands. Hum. Genet. 56:293298.

Yunis JJ, and Prakash O (1982) The origin of man: A chromosomal pictorial legacy. Science 215:15251530 . 